04

\title{
Зависимость потенциала пробоя от скорости роста напряжения в длинной разрядной трубке при низком давлении
}

\author{
() Ю.З. Ионих, А.В. Мещанов, Д.О. Иванов \\ Санкт-Петербургский государственный университет, \\ 199034 Санкт-Петербург, Россия \\ e-mail: y.ionikh@spbu.ru
}

Поступило в Редакцию 7 ноября 2018 г.

В окончательной редакции 7 ноября 2018 г.

Принято к публикации 22 января 2019 г.

Измерено динамическое напряжение пробоя в длинной $(80 \mathrm{~cm})$ разрядной трубке в неоне, аргоне, их смеси и в аргоне с парами ртути при давлениях 80-400 Ра в широком диапазоне изменения скорости роста анодного напряжения $\left(d U / d t \sim 10^{-4}-10^{2} \mathrm{kV} / \mathrm{ms}\right)$. Обнаружен немонотонный ход зависимости потенциала пробоя от $d U / d t$ с минимумом в области $0.1-10 \mathrm{kV} / \mathrm{ms}$. Рост пробойного потенциала в области больших $d U / d t$ связан с наличием времени запаздывания пробоя. Увеличение потенциала пробоя в области низких скоростей роста напряжения предположительно обусловлено спецификой пробоя в длинных трубках и связано с накоплением поверхностного заряда на стенке трубки. Заряд уменьшает разность потенциалов между анодом и стенкой и затрудняет первичной пробой между ними. Результаты дополнительных экспериментов с импульсами, наложенными на постоянную подложку, подтверждают возможность такого объяснения.

DOI: $10.21883 /$ JTF.2019.07.47789.387-18

\section{Введение}

В светотехнике, в газоразрядных лазерах, в световой рекламе, а также при исследовании плазмы используются так называемые длинные разрядные трубки. Длинной называется трубка, у которой расстояние между электродами намного больше ее диаметра. Зажигание разряда (пробой) в таких трубках происходит не так, как в коротких промежутках с большой поверхностью электродов, где электрическое поле до пробоя близко к однородному и где при низком давлении реализуются таунсендовский (лавинный) механизм. Пробой в длинной трубке предваряется распространением одной или нескольких волн ионизации. Волна ионизации (ВИ) это локализованная область высокого градиента потенциала электрического поля, движущаяся вдоль трубки от высоковольтного электрода (ВВЭ) к низковольтному. Волна создает начальную плазму, которая затем развивается в плазму разряда. ВИ, в свою очередь, возникает при первичном пробое между высоковольтным электродом и стенкой трубки. Такой сценарий был впервые описан в работе [1]. Прохождение предпробойной ВИ при зажигании тлеющего разряда низкого давления было подтверждено в последующих работах (см. работу [2] и приведенные в ней ссылки). Как правило, ВИ регистрируется по ее излучению. Отметим, что здесь речь идет о волнах со скоростью распространения порядка $10^{5}-10^{7} \mathrm{~cm} / \mathrm{s}$, что в соответствии с классификацией [3] определяется как медленные ВИ.

При движении ВИ от высоковольтного электрода к заземленному происходит зарядка стенки трубки. Стенка заряжается током, который протекает через плазменный след, оставленный волной, и далее через емкость трубка-земля. Плотность поверхностного заряда измерялась в работах [4,5], а потенциал стенки в работах $[5,6]$. Эти измерения проводились с помощью емкостного (электростатического) зонда. В работе [1] такой же зонд применялся для регистрации движения фронта ионизации. Считается, что зарядка стенки играет очень важную роль в процессе развития пробоя. Поверхностный заряд стенки определяет конфигурацию электрического поля в объеме в каждый момент времени и влияет на продвижение ВИ. В некоторых условиях он может, например, препятствовать пробою [5]. По мнению авторов работ $[4,5,7]$ наличие на стенке заряда, оставшегося после предыдущего импульса, может создавать неоднозначные начальные условия для пробоя. Для исключения этого в работах по исследованию пробойных процессов в промежутке между импульсами зажигают кратковременный „сбрасывающий (resetting) разряд, который должен снять заряд с поверхности $[4,5,7]$. Насколько эффективно это происходит, неизвестно. Авторы [5] предполагают, что заряд не удаляется полностью, но пробой все же становится надежным и воспроизводимым.

В недавней работе [8] процесс зарядки стенки при движении ВИ регистрировался путем измерения тока ВВЭ; интегрирование этого тока по всему времени прохождения волны дает полную величину заряда. В этой работе изучался пробой в трубке с отсоединенным от цепи питания низковольтным электродом („одноэлектродный разряд“ [9]). В такой схеме пробой всего разрядного промежутка невозможен. После того как ВИ проходит по всей длине трубки, ток в цепи ВВЭ 
прекращается, а стенка остается заряженной все время, пока на электроде поддерживается высокий потенциал. Разрядка стенки происходит после окончания импульса напряжения, когда ВВЭ заземляется. При этом, как показано в [8], наблюдается пробой между заряженной стенкой и электродом; он аналогичен тому первичному пробою, который происходил в начале импульса, и также сопровождается волной ионизации, но с противоположным по знаку током („обратный пробой“). Обратный пробой происходит не сразу после окончания импульса, а через некоторый случайный промежуток времени (время запаздывания пробоя). Если до истечения этого промежутка на электрод снова подать высокое напряжение, то новый первичный пробой не возникает. В [8] это объяснялось тем, что пока стенка заряжена ее потенциал остается высоким, и при подаче напряжения на электрод разность потенциалов между ним и стенкой близка к нулю. Можно предположить, что пробой можно все же осуществить, если увеличить потенциал ВВЭ. Другими словами, можно ожидать, что наличие заряда стенки в момент пробоя может привести к увеличению пробойного потенциала.

По умолчанию под пробойным потенциалом понимается обычно так называемый статический потенциал (или напряжение) пробоя $U_{S}$. Это минимальное напряжение, при котором поддерживается самостоятельная форма разряда [10,11]. Если, начиная с нуля, непрерывно повышать межэлектродное напряжение $U$, то значение $U_{b}$, при котором произойдет пробой, будет, вообще говоря, превышать $U_{S}$. Это связано с запаздыванием пробоя относительно того момента, когда $U=U_{S}$, в результате чего

$$
U_{b}=U_{S}+(d U / d t) t_{d}
$$

где $t_{d}$ - время запаздывания пробоя. Поэтому для измерения $U_{S}$ определяют потенциал пробоя $U_{b}$ при различной скорости роста напряжения и затем находят $U_{S}$ экстраполяцией $d U / d t$ к нулевому значению $[11,12]$. Рассматривая широкий интервал изменения величины $d U / d t$, можно выделить два ее предельных значения:

1) Очень медленный рост напряжения, когда

$$
(d U / d t) t_{d} \ll U_{S}
$$

В этом случае $U_{b}=U_{S}$.

2) Импульс напряжения с очень крутым передним фронтом, когда

$$
(d U / d t) t_{d} \gg U_{0},
$$

где $U_{0}-$ амплитуда импульса (предполагается, что $\left.U_{0}>U_{S}\right)$. В этом случае $U_{b}=U_{0}$.

В обоих предельных случаях напряжение пробоя фиксировано. В общем случае это не так. Время запаздывания пробоя может быть представлено [13] как сумма двух величин: статистического времени и времени формирования (formative time) разряда

$$
t_{d}=t_{s}+t_{f} \text {. }
$$

Статистическое время запаздывания $t_{s}$ есть время, в течение которого в промежутке появляется электрон, инициирующий лавину, развитие которой приводит к пробою („эффективный электрон“ [11]); эта величина стохастическая и может флуктуировать в широком интервале. Время формирования $t_{f}-$ это интервал от момента появления такого электрона до скачка тока в разрядной цепи, т. е. до пробоя. Стохастичность величины $t_{s}$ приводит к флуктуациям полного времени запаздывания $t_{d}$ (в неравенствах (2), (3) под величиной $t_{d}$ следует понимать ее усредненные значения). В свою очередь, разброс $t_{d}$ приводит, согласно соотношению (1), к разбросу значений пробойного напряжения $U_{b}$, для которого вводят в этом случае термин ,динамическое напряжение пробоя“ (иногда этот термин используют для величины, полученной усреднением по большому числу пробоев). Разброс времени запаздывания и пробойного напряжения может быть уменьшен или практически устранен дополнительным источником инициирующих электронов. Действительно, согласно Лауэ [14], $\overline{t_{s}}=1 / z$, где $z$ - число эффективных электронов, появившихся в единицу времени. Поэтому увеличение $z$ приводит к уменьшению статистического времени и его флуктуаций. Источником электронов может быть термо- или фотоэмиссия электронов с катода, достаточно интенсивная, чтобы среднее значение $t_{s}$ стало намного меньше $t_{f}$ [15].

Сказанное выше относится, строго говоря, к случаю лавинного механизма пробоя, т. е. пробоя в полях, близких к однородному. Как отмечалось выше, в длинных трубках этот механизм не реализуется. Считается, однако, что первичный пробой между ВВЭ и ближайшим участком стенки трубки происходит именно по такому механизму [5], поэтому для него такой подход справедлив. Специфика длинной трубки проявляется в том, что момент пробоя всего промежутка не совпадает с моментом первичного пробоя, а отстоит от него на время прохождения волны (или волн) ионизации. Поэтому полное время запаздывания пробоя увеличивается на эту величину

$$
t_{d}=t_{s}+t_{f}+t_{w}=t_{s}+t_{f w},
$$

где $t_{w}$ - время движения ВИ, а величину

$$
t_{f w}=t_{f}+t_{w}
$$

можно рассматривать как время формирования для всего разрядного промежутка. Как и в случае короткого разрядного промежутка, время $t_{s}$ и тем самым разброс значений $t_{d}$ могут быть подавлены с помощью дополнительного источника электронов, инициирующих первичный пробой. При отрицательном потенциале ВВЭ это может быть термоэмиссия с нагреваемого катода [4-7]. При положительном потенциале ВВЭ такой эффект можно достичь освещением прианодной области трубки излучением с длиной волны короче $\sim 500 \mathrm{~nm}[2,16,17]$. Механизм такого воздействия - это предположительно фотодесорбция электронов, адсорбированных на поверхности стенки $[2,16]$. В случае импульса с фронтом конеч- 
ной ширины, подавление $t_{s}$ и флуктуаций $t_{d}$ приводит к исчезновению разброса $U_{b}$.

В любом случае, однако, время запаздывания влияет на величину динамического напряжения пробоя. Как следует из (1), из-за него должен наблюдаться монотонный рост $U_{b}$ при увеличении $d U / d t$. Именно такая зависимость получается при исследовании пробоя в однородных полях $[11,12,18,19]$. Для пробоя в длинных трубках данные о зависимости $U_{b}$ от $d U / d t$ отсутствуют (в часто цитируемой работе [20] измерения $U_{b}$ проводятся для фиксированного значения $d U / d t)$. В настоящей работе были проведены измерения пробойного напряжения в длинной трубке в широкой (охватывающей более 6 порядков величины) области изменения $d U / d t$. Оказалось, что реальное поведение этой зависимости существенно отличается от предсказываемого формулой (1). Полученным при этом результатам и их возможной трактовке посвящена настоящая работа.

\section{Экспериментальная установка}

Использовались стеклянные цилиндрические разрядные трубки с расстоянием между электродами $75-80 \mathrm{~cm}$ и внутренним диаметром 15-28 mm. Цилиндрические электроды диаметром от 10 до $25 \mathrm{~mm}$ и длиной 25-50 mm изготовлены из алюминия, железа с никелевым покрытием или тантала. Использовались также подогреваемые электроды. Трубки были как прокачиваемые, так и отпаянные. Применялись чистые (99.98\%) газы - неон, аргон, их смесь, а также смесь аргона с парами ртути. Один из электродов трубки (катод) соединялся с землей через низкоомное сопротивление (оно использовалось для регистрации тока в разрядной цепи), на другой подавались положительные импульсы амплитудой до $10 \mathrm{kV}$ и длительностью, как правило, $10 \mathrm{~ms}$. Частота повторения импульсов была низкой порядка $0.1 \mathrm{~Hz}$. Это было сделано для устранения влияния на пробой возбужденных и заряженных частиц, наработанных предыдущим импульсом („эффект памяти разрядного промежутка“" $[11,20])$. Импульсы имели линейно-растущий фронт варьируемой крутизны, либо были прямоугольной формы с длительностью фронта $\sim 1 \mu \mathrm{s}$. Ток разряда, возникавшего после пробоя, ограничивался балластным сопротивлением на уровне порядка $10 \mathrm{~mA}$.

Динамическое напряжение пробоя $U_{b}$ определялось из осциллограмм анодного напряжения. В этом случае импульсы имели линейно-растущий фронт, и в момент пробоя происходил резкий сброс напряжения. Его значение в точке максимума считалось равным $U_{b}$. Использовались цифровые осциллографы с высоковольтным щупом Tektronix P6013A на входе. Измерения проводились при освещении трубки стандартной люминесцентной лампой мощностью $30 \mathrm{~W}$, находившейся на расстоянии $0.5 \mathrm{~m}$. Такого освещения было, как правило, достаточно, чтобы существенно снизить флуктуации $U_{b}$. Это означало, что в равенстве $(5) t_{s} \ll t_{f w}$ и $t_{d}=t_{f w}$. Величина $U_{b}$ принимает при этом, очевидно, наименьшее для данной крутизны фронта $d U / d t$ значение. Оставшийся разброс уменьшался усреднением по 30-50 измерениям (при минимальных скоростях роста напряжения - по 5 измерениям). Измерялось также время запаздывания пробоя $t_{d}$. В этом случае импульсы были прямоугольные, и в качестве $t_{d}$ брался промежуток между передним фронтом импульса и моментом сброса напряжения. Более детальное описание установки приведено в рабо$\operatorname{Tax}[2,21]$.

\section{Экспериментальные результаты и их обсуждение}

Измерение потенциала пробоя $U_{b}$ проводилось при подаче на анод трубки напряжения с линейно-растущим фронтом варьируемого наклона $d U / d t$. На рис. 1 приведена зависимость напряжения пробоя от крутизны фронта импульса в отпаянной трубке диаметром $15 \mathrm{~mm}$ в неоне при давлении $80 \mathrm{~Pa}$ и частоте повторения импульсов $0.5 \mathrm{~Hz}$. Размер экспериментальных точек на графике примерно отражает величину разброса результатов. Значение $d U / d t$ меняется в диапазоне $0.7 \cdot 10^{-4}-1.3 \cdot 10^{2} \mathrm{kV} / \mathrm{ms}\left(0.7 \cdot 10^{2}-1.3 \cdot 10^{8} \mathrm{~V} / \mathrm{s}\right)$. Видно, что зависимость $U_{b}$ от $d U / d t$ существенно немонотонная. Ожидаемый рост наблюдается только при $d U / d t>2 \mathrm{kV} / \mathrm{ms}$. Врезка на рисунке показывает, что на небольшом участке от 2 до $\sim 20 \mathrm{kV} / \mathrm{ms}$ зависимость имеет линейный характер, т.е. формально описывается формулой (1) с параметрами $U_{S}=1.18 \mathrm{kV}$, $t_{d}=14 \mu \mathrm{s}$. Эти значения близки к результатам работы [16], где изучался пробой в тех же условиях. При $d U / d t>20 \mathrm{kV} / \mathrm{ms}$ экспериментальные точки системати-

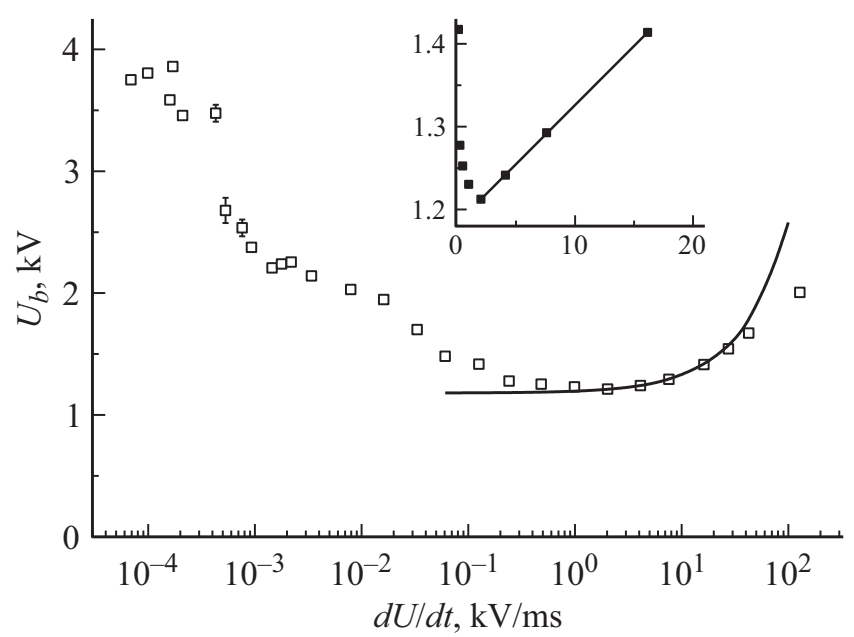

Рис. 1. Зависимость потенциала пробоя от скорости роста анодного напряжения в неоне при давлении 80 Ра. Точки эксперимент, кривая - линейная зависимость (формула (1)). На врезке - область $d U / d t<16 \mathrm{kV} / \mathrm{ms}$. 


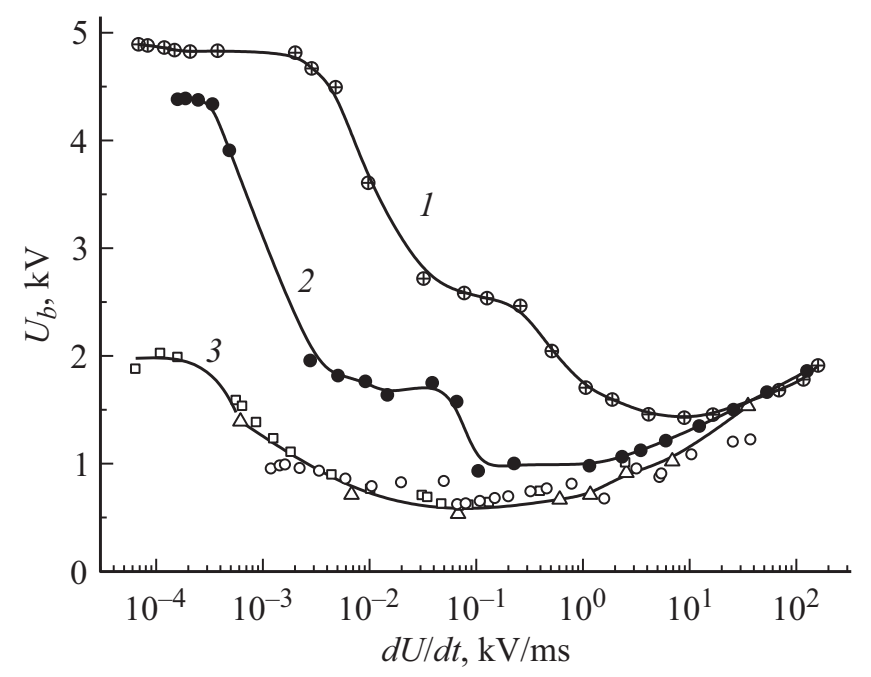

Рис. 2. Зависимость потенциала пробоя от скорости роста анодного напряжения в неоне (1), смеси $\mathrm{Ne}-\mathrm{Ar}, 3: 1$ (2) и в аргоне с парами ртути $\left(p_{\mathrm{Hg}}=0.1 \mathrm{~Pa}\right)$. Полное давление газа $400 \mathrm{~Pa}$.

чески уходят от линейной зависимости, изображаемой кривой на рис. 1. Это можно объяснить тем, что время запаздывания пробоя уменьшается с ростом напряжения как для коротких разрядных промежутков [11,22], так и длинных трубок [16]. Поэтому при изменении $d U / d t$ в широких пределах рост $U_{b}$ оказывается медленнее линейного [12,18,19,23] (в частности, в [18,23] $U_{b}$ растет как $\left.(d U / d t)^{1 / 2}\right)$. Во всех этих работах исследовался пробой коротких промежутков. Для длинных трубок аналогичных данных нет. Тем не менее можно достаточно уверенно утверждать, что правая ветвь кривой рис. 1 обусловлена ростом второго слагаемого равенства (1) при увеличении крутизны фронта $d U / d t$.

Труднее объяснить рост пробойного напряжения при уменьшении крутизны фронта, когда она меньше $\sim 1 \mathrm{kV} / \mathrm{ms}$. Для коротких промежутков подобного не наблюдалось во всем исследованном диапазоне $d U / d t$, вплоть до очень малых значений: $10^{-2} \mathrm{~V} / \mathrm{s}$ для пробоя в азоте [12] и $10^{-1} \mathrm{~V} / \mathrm{s}$ в неоне [19]. Эксперименты в длинных трубках нам не известны.

На рис. 2 представлены результаты измерений для пробоя в неоне $(1)$, в смеси $\mathrm{Ne}-\mathrm{Ar}(3: 1)$ (2) и в смеси $\mathrm{Ar}-\mathrm{Hg}$ (парциальное давление паров ртути 0.1 $\mathrm{Pa}$ ) (3) при полном давлении газа $400 \mathrm{~Pa}$. В первых двух случаях использовалась описанная выше трубка диаметром $15 \mathrm{~mm}$, в третьем - бактерицидная ртутная лампа диаметром $25 \mathrm{~mm}$. Немонотонный характер получается для всех кривых. Кривые 1 и 2 имеют дополнительную структуру в промежуточной области $d U / d t$. Для всех кривых также отчетливо проявляется выход на постоянный уровень при минимальных значениях аргумента. Тенденция к такому насыщению видна и для экспериментальных точек на рис. 1.
Как уже отмечалось, при пробое коротких разрядных промежутков закономерности, похожие на полученные в настоящей работе, не наблюдаются. Имеет смысл поэтому обратиться к различию механизмов пробоя в коротких и длинных промежутках. В первом случае процесс пробоя начинается с размножения электронов в объеме газа в электрическом поле между электродами. Во втором же случае начальная стадия - это первичный пробой между высоковольтным электродом и ближайшим участком стенки. При стандартных условиях зажигания разряда до подачи напряжения на ВВЭ вся трубка вместе с электродами находится под нулевым потенциалом относительно земли. При подаче напряжения $U$ на ВВЭ все это напряжение оказывается приложенным между электродом и стенкой, и возможность реализации первичного пробоя определяется величиной $U$ (в частности, необходимое условие пробоя - это $\left.U>U_{S}\right)$. Представим теперь, что за некоторое время до включения напряжения $U$ на ВВЭ подан потенциал $u_{0}$. Если $u_{0}<U_{S}$, то этот потенциал не произведет пробоя. Но он, благодаря конечной проводимости поверхности стекла и наличию на ней адсорбированных электронов, может повысить потенциал стенки вблизи ВВЭ, возможно, вплоть до величины $u_{0}$. Тогда при подаче на ВВЭ напряжения $U$ разность потенциалов между электродом и стенкой будет меньше $U$, и теперь для пробоя требуется выполнение неравенства $U-u_{0}>U_{S}$. Таким образом, пробойное напряжение увеличится на величину $u_{0}$ (или, возможно, меньшую, если потенциал стенки не достигает $\left.u_{0}\right)$.

Для проверки этого предположения был проведен эксперимент, в котором импульсы с линейно-растущим фронтом накладывались на постоянное напряжение $u_{0}$ и измерялось напряжение пробоя. Результаты демонстрирует график рис. 3, на котором показано пробойное напряжение как функция $d U / d t$ при наличии подложки (2)

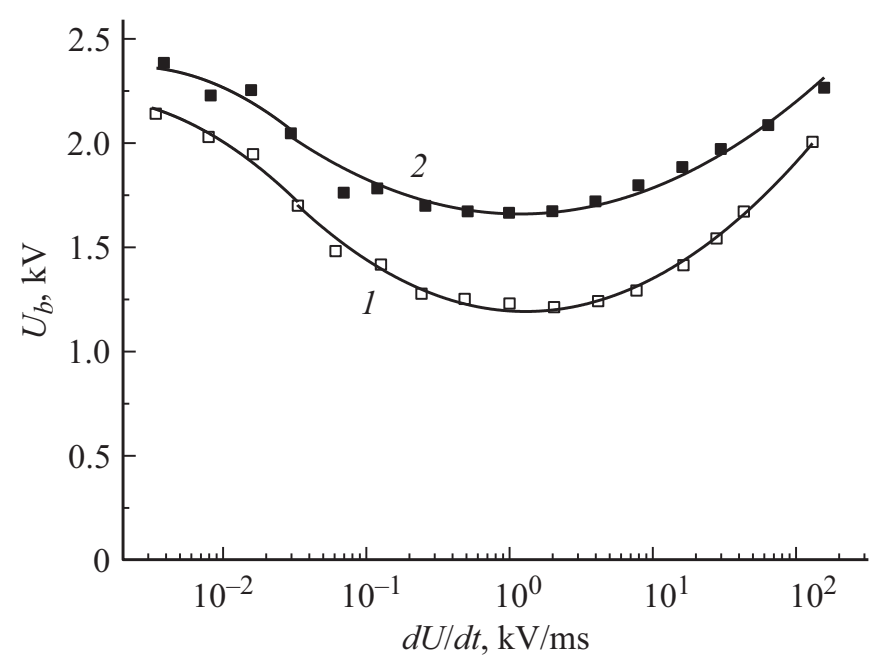

Рис. 3. Зависимость потенциала пробоя от скорости роста анодного напряжения в неоне при давлении 80 Ра при наличии постоянной подложки $u_{0}=500 \mathrm{~V}(2)$ и без нее $(1)$. 


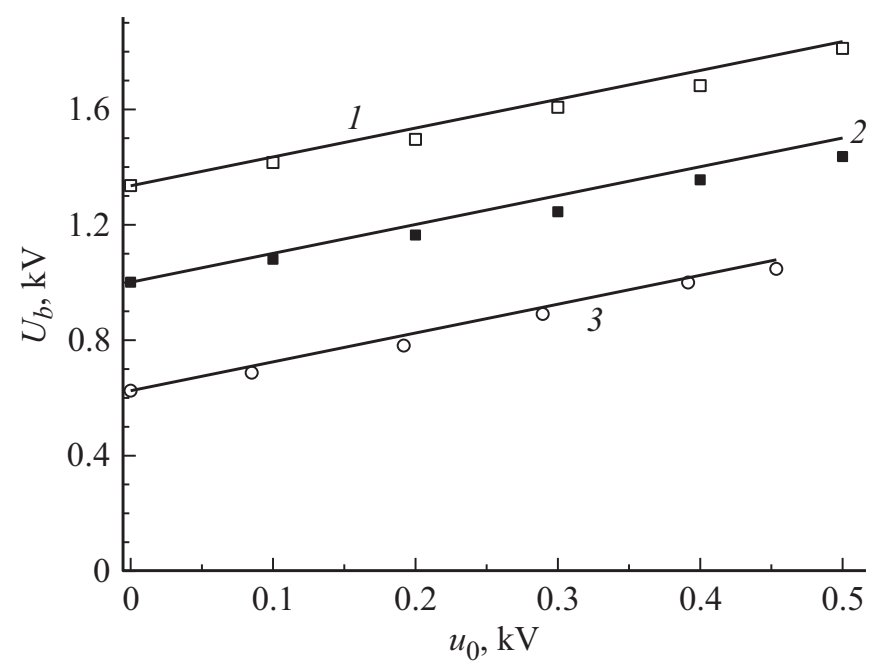

Рис. 4. Зависимость напряжения пробоя от величины постоянной подложки в $\mathrm{Ne}$ при давлении $80 \mathrm{~Pa}, d U / d t=1 \mathrm{kV} / \mathrm{ms}(1)$; в смеси $\mathrm{Ne}-\mathrm{Ar}(3: 1), 400 \mathrm{~Pa}, 1 \mathrm{kV} / \mathrm{ms}$ (2) и в $\mathrm{Ar}, 133 \mathrm{~Pa}$, $0.05 \mathrm{kV} / \mathrm{ms}$ (3). Прямые - расчет по формуле (7).

и без нее $(1)$. Воспроизведены условия рис. 1, величина постоянной положки $u_{0}=500 \mathrm{~V}$. Видно, что в широком диапазоне $d U / d t$ имеется заметное превышение $U_{b}$ над $U_{b}^{0}$, причем для $d U / d t \sim 1 \mathrm{kV} / \mathrm{ms}$ их разность близка к высоте подложки $u_{0}$.

В другом эксперименте напряжение пробоя измерялось для различных уровней подложки $u_{0}$, но при фиксированном наклоне. Полученные результаты показаны точками на рис. 4 для условий: $1-$ неон, давление $80 \mathrm{~Pa}$; 2 - смесь $\mathrm{Ne}-\mathrm{Ar}$ (3:1), $400 \mathrm{~Pa} ; 3$ - аргон, $133 \mathrm{~Pa}$. В первых двух случаях использовалась отпаянная трубка диаметром $15 \mathrm{~mm}$; в третьем - проточная трубка диаметром $28 \mathrm{~mm}$. Прямыми на этом графике изображены функции

$$
U_{b}=U_{b}^{0}+u_{0},
$$

где $U_{b}^{0}-$ значение $U_{b}$ при $u_{0}=0$. Видно, что во всех случаях пробойное напряжение увеличивается на величину, близкую к $u_{0}$.

Альтернативный подход к наблюдению этого эффекта состоит в измерении времени запаздывания пробоя $t_{d}$. В настоящей работе время запаздывания и его компоненты - статистическое время $t_{s}$ и время формирования $t_{f w}(5),(6)$ - измерялись для импульсов напряжения прямоугольной формы. В этом случае при достаточной для пробоя амплитуде импульса $U_{0}$ все пробои происходят при этом напряжении, т.е. $U_{b}=U_{0}$, но при случайном времени запаздывания. Изучалась серия из 300 импульсов, для каждого из которого измерялось $t_{d}$. Разделение его на компоненты $t_{s}$ и $t_{f w}$ производилось с помощью построения графиков распределения числа пробоев по времени запаздывания,

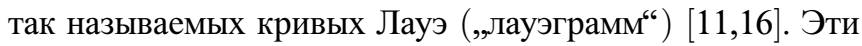
измерения велись в темноте, так как при освещении трубки статистическое время оказывалось слишком коротким, что бы его можно было надежно измерить.

На рис. 5, $a$ показаны результаты измерений для случая, когда импульсы амплитудой $U_{0}=1.5$ и $2 \mathrm{kV}$ накладывались на постоянное напряжение $u_{0}=0-500 \mathrm{~V}$. Наблюдается рост значений $t_{s}$ и $t_{f w}$ при увеличении $u_{0}$ (в том числе и $t_{f w}$ для $2 \mathrm{kV}$, где эта зависимость выражена слабо). Для объяснения этих результатов надо учесть, что все компоненты времени запаздывания растут при уменьшении амплитуды импульса $[11,16]$. В данном случае амплитуда $U_{0}$ постоянна. Но, как следовало из графиков рис. 3,4 , наличие постоянной подложки напряжения снижает действующий потенциал
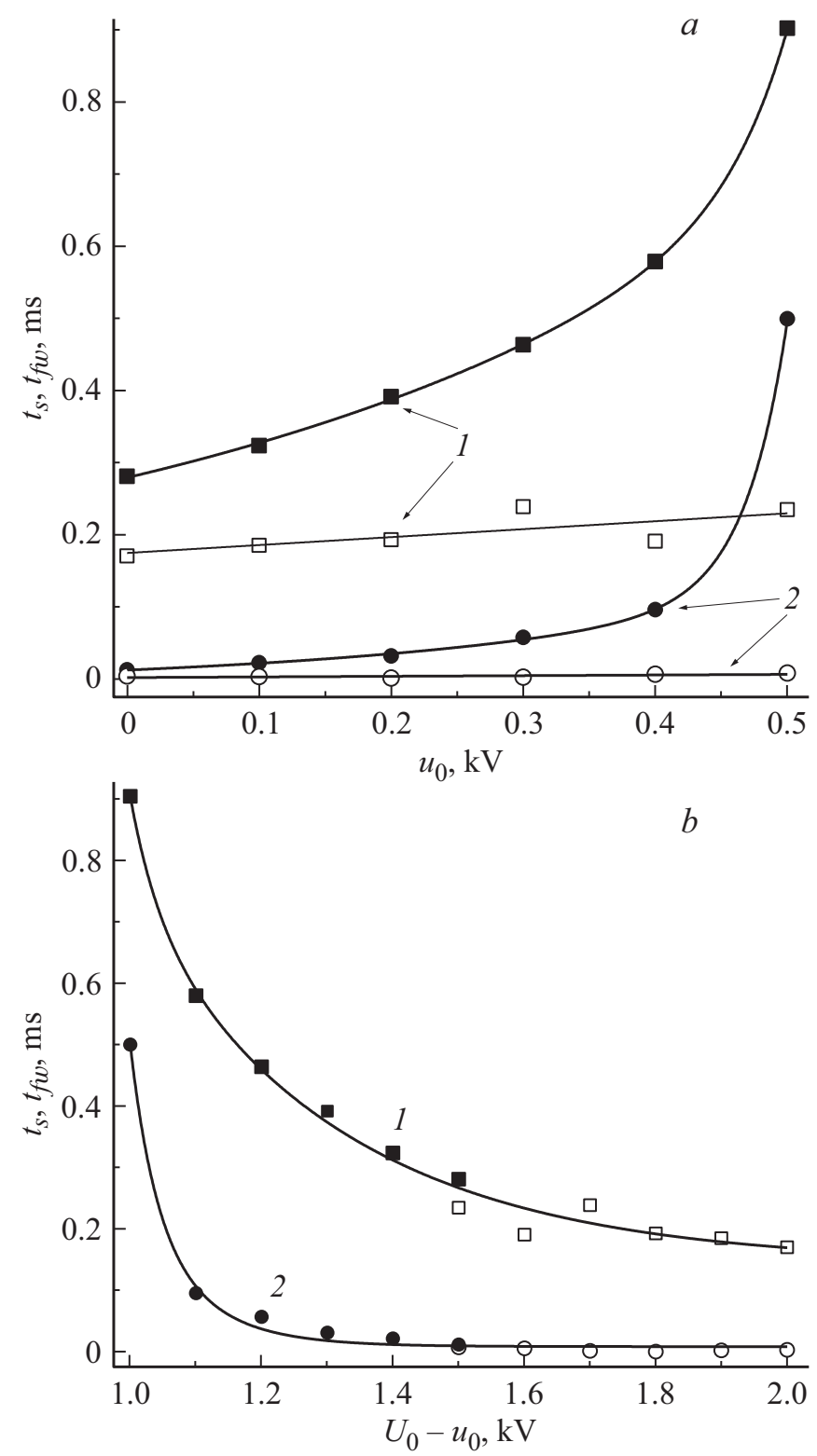

Рис. 5. Статистическое время запаздывания (1) и время формирования (2) в неоне при $p=80 \mathrm{~Pa}$ в зависимости от напряжения постоянной подложки $(a)$ и от разности между амплитудой импульса анодного напряжения и величиной положки $(b)$. Черные точки $-U_{0}=1.5 \mathrm{kV}$, белые $-2 \mathrm{kV}$. 


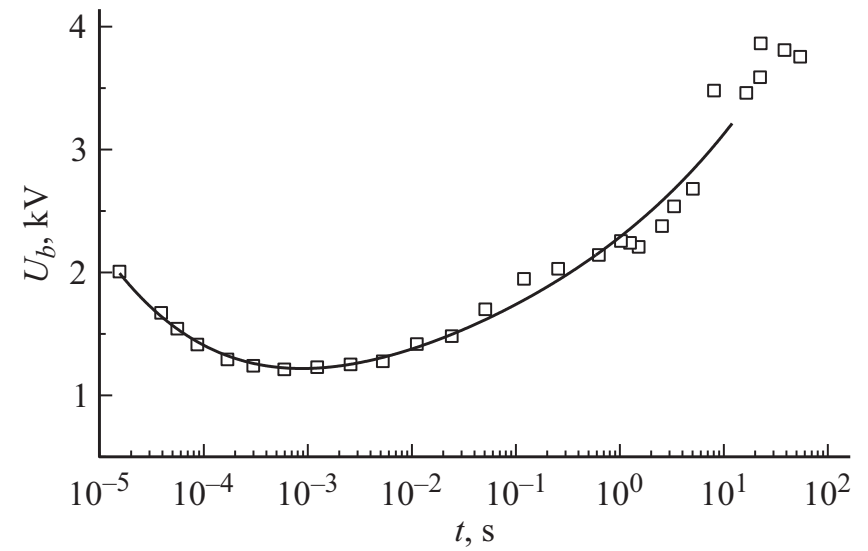

Pис. 6. Пробойный потенциал как функция времени от начала роста напряжения до пробоя. Неон, $p=80 \mathrm{~Pa}$.

на величину, близкую к $u_{0}$. Увеличение $u_{0}$ приводит к уменьшению этого потенциала, что и должно удлинять запаздывание. Отсюда также следует, что именно разность $U_{0}-u_{0}$ должна определять характеристики пробоя, в том числе время запаздывания. На рис. $5, b$ те же данные представлены как функции величины $U_{0}-u_{0}$. В этом случае действительно оба набора точек для $t_{s}$ укладываются на общую кривую, и это же справедливо для $t_{f w}$.

Вернемся к результатам, представленным на рис. 1, 2, и рассмотрим область $d U / d t$ слева от минимума. Для этой области промежуток времени от начала роста напряжения до пробоя составляет от $\sim 10 \mathrm{~ms}$ до почти $100 \mathrm{~s}$ (рис. 6). Можно предположить, что, во-первых, в течение этого времени потенциал стенки в прианодной области также растет, хотя и медленнее потенциала самого анода, и, во-вторых, чем меньше скорость роста напряжения, тем меньше отставание потенциала стенки от потенциала анода. Пробой произойдет тогда, когда разность потенциалов между ними превысит $U_{S}$ (вклад второго слагаемого в равенстве (1) при малых $d U / d t$ незначителен). В результате с уменьшением $d U / d t$ напряжение пробоя растет. При этом фактическое значение статического напряжения пробоя оказывается больше номинальной величины $U_{S}$, отвечающей отсутствию заряда на стенке. Таким образом, в отличие от правой ветви кривых рис. 1, 2 левая ветвь может быть качественно объяснена ростом первого слагаемого равенства (1) при уменьшении крутизны фронта.

Очевидно, что предлагаемое объяснение носит достаточно гипотетический характер. Для его подтверждения необходимо проведение дополнительных исследований, дающих информацию о поверхностном заряде стенки, процессах его появления, миграции и стекания. Как упоминалось во Введении, для длинных трубок имеются данные только о величине заряда $[4,5,8]$ и потенциала $[5,6]$, возникающих при прохождении предпробойной ВИ. Есть также работы [24,25], в которых из сравнения модельных расчетов с экспериментом по- лучены оценки плотности электронов на стенке, контактирующей с разрядной плазмой. Более развернутая информация имеется о заряде на поверхности диэлектрика в барьерных разрядах атмосферного давления (DBD). Измерена не только величина заряда, но и характерное время его стекания, достигающее десятков минут [26-28], и время миграции вдоль поверхности ( 10s [29]). Для условий, характерных для зажигания тлеющего разряда в трубках, таких данных пока нет.

Представляет интерес тот факт, что немонотонная зависимость потенциала пробоя от длительности фронта импульса, т.е. от скорости роста напряжения, наблюдается и в совершенно иных условиях - при пробое длинных промежутков в воздухе в условиях атмосферного давления и резко неоднородного поля (см. [30] и приведенные там ссылки). Так, в [31], где исследовался пробой между стержнем и плоскостью с расстоянием между ними 1-3 m, эти зависимости похожи на кривые рис. 6, при том, что параметры, конечно, отличаются очень сильно. В частности, минимальные напряжения пробоя составляют 400-900 kV. Интересно, что авторы дают объяснение этим зависимостям, сходные с предлагаемыми в настоящей работе. Рост $U_{b}$ в сторону больших $d U / d t$ они относят за счет конечного времени предпробойных процессов (формирования предпробойной короны и лидера), т. е. своего рода времени запаздывания пробоя. При малых же $d U / d t$ пробой затруднен накоплением объемного заряда в приэлектродной области, уменьшающего напряженность поля в ней. В нашем случае эту роль может играть поверхностный заряд стенки.

\section{Заключение}

Измерено напряжение пробоя в длинной $(80 \mathrm{~cm})$ разрядной трубке в неоне, аргоне, их смеси и в аргоне с парами ртути при полном давлении $80-400 \mathrm{~Pa}$ в широкой области изменения скорости роста анодного напряжения $\left(d U / d t \sim 10^{-4}-10^{2} \mathrm{kV} / \mathrm{ms}\right)$. Обнаружен немонотонный ход зависимости потенциала пробоя от $d U / d t$, с минимумом в области $0.1-10 \mathrm{kV} / \mathrm{ms}$. Напряжение пробоя при минимальных скоростях роста до 3 раз превышает его наименьшее значение. Рост пробойного потенциала с увеличением $d U / d t$, характерный и для пробоя коротких промежутков, обусловлен сушествованием времени запаздывания пробоя. Рост напряжения пробоя при уменьшении $d U / d t$ может быть связан со спецификой пробоя в длинных трубках, начальной фазой которого является первичный пробой между высоковольтным электродом и ближайшим участком стенки трубки. Можно предположить, что при медленном росте анодного напряжения на внутренней поверхности стенки успевает накопиться заряд, который снижает разность потенциалов между электродом и стенкой, и этим затрудняет пробой. Такую возможность подтверждают эксперименты с импульсом, наложенным на постоянную подложку. Потенциал про- 
боя в этом случае возрастает на величину, близкую к напряжению подложки. При пробое прямоугольными импульсами наличие постоянной подложки увеличивает время запаздывания пробоя до значения, отвечающего разности между амплитудой импульса и напряжением подложки.

\section{Финансирование работы}

Работа выполнена при финансовой поддержке Российского фонда фундаментальных исследований (проект 19-02-00288).

\section{Список литературы}

[1] Недоспасов А.В., Новик А.Е. // ЖТФ. 1960. Т. 30. С. 1329.

[2] Meshchanov A.V., Ivanov D.O., Ionikh Y.Z., Shishpanov A.I. // J. Phys. D: Appl. Phys. 2018. Vol. 51. P. 335202.

[3] Василяк Л.М., Асиновский Э.И., Самойлов И.С. Энциклопедия низкотемпературной плазмы. Вводный том. Кн. II / Под ред. В.Е. Фортова. М.: Наука, 2000. С. 225.

[4] Horstman R.E., Lansink F.M.O. // J. Phys. D: Appl. Phys. 1988. Vol. 21. P. 1130.

[5] Gendre M.F., Haverlag M., Kroesen G.M.W. // J. Phys. D: Appl. Phys. 2010. Vol. 43. P. 234004.

[6] Gendre M.F., Bowden M.D., van den Nieuwenhuizen H.C.M., Haverlag M., Gielen J.W.A.M., Kroesen G.M.W. // IEEE Trans. Plasma Sci. 2005. Vol. 33. P. 262.

[7] Gendre M.F., Bowden M.D., Haverlag H., van den Nieuwenhuizen H.C.M., Gielen J., Kroesen G.M.W. // Proc.Frontiers in Low Temperature Plasma Diagnostics V: Proc. of International Workshop (Villaggio: Cardigliano). 2003. P. 295.

[8] Калинин С.А., Капитонова М.А., Матвеев Р.М., Мещанов А.В., Ионих Ю.З. // Физика плазмы. 2018. Т. 44. № 11. C. $870-880$.

[9] Туницкий Л.Н., Игнашев А.И. // Светотехника. 1955. № 2. C. 23.

[10] Райзер Ю.П. Физика газового разряда. М.: Наука, 1992. $536 \mathrm{c}$.

[11] Pejović M.M., Ristić G.S., Karamarković J.P. // J. Phys. D: Appl. Phys. 2002. Vol. 35. P. R91-103.

[12] Pejović M.M., Filipović R.D. // Int. J. Electron. 1989. Vol. 67. P. 251.

[13] Мик Джс., Крэгс Дж. Электрический пробой в газах. М.: ИЛ, 1960.

[14] Laue M. von. // Ann. D. Phys. 1925. Vol. 76. S. 261.

[15] Королев Ю.Д., Месяи Г.А. Физика импульсного пробоя газов. М.: Наука, 1991. 224 с.

[16] Shishpanov A.I., Meshchanov A.V., Kalinin S.A., Ionikh Y.Z. // Plasma Sources Sci. Technol. 2017. Vol. 26. P. 065017.

[17] Nie L., Xian Y., Lu X., Ostrikov K. // Phys. Plasmas. 2017. Vol. 24. P. 043502.

[18] Marković V.Lj., Goci]'c S.R., Stamenković S.N., Petrović Z.Lj. // Eur. Phys. J. Appl. Phys. 2005. Vol. 30. P. 51.

[19] Maluckov Č.A., Radović M.K. // IEEE Trans. Plasma Sci. 2002. Vol. 30. P. 1597.

[20] Lisovskiy V.A., Yakovin S.D., Yegorenkov V.D. // J. Phys. D: Appl. Phys. 2000. Vol. 33. P. 2722.
[21] Шишпанов А.И., Ионих Ю.З., Мещанов А.В., Дятко Н.А. // Физика плазмы. 2014. Т. 40. С. 548.

[22] Radović M.K., Maluckov Č.A., Karamarković J.P., Rančev S.A., Mitić S.D. // Romanian Rep. Phys. 2014. Vol. 66. P. 472.

[23] Marković V.Lj., Stamenković S.N., Gocić S.R. // Rev. Sci. Instr. 2006. Vol. 77. P. 096104.

[24] Uhrlandt D., Schmidt M., Behnke J.F., Bindemann T. // J. Phys. D: Appl. Phys. 2000. Vol. 33. P. 2475.

[25] Heinisch R.L., Bronold F.X., Fehske H. // Phys. Rev. B. 2012. Vol. 85. P. 075323.

[26] Li M., Li C., Zhan H., Xu J., Wang X. // Appl. Phys. Lett. 2008. Vol. 92. P. 031503.

[27] Ambrico P.F., Ambrico M., Schiavull L., De Benedictis S. // J. Phys. D: Appl. Phys. 2014. Vol. 47. P. 305201.

[28] Tschiersch R., Bogaczyk M., Wagner H-E. // J. Phys. D: Appl. Phys. 2014. Vol. 47. P. 365204.

[29] Wild R., Benduhn J., Stollenwerk L. // J. Phys. D: Appl. Phys. 2014. Vol. 47. P. 435204.

[30] Базелян Э.М., Райзер Ю.П. Искровой разряд. М.: МФТИ, 1997.

[31] Стекольников И.С., Браго Е.Н., Базелян Э.М. // ДАН CCCP. 1960. T. 133. С. 550. 